\title{
Laparoscopic Cholecystectomy with Low Tension Pneumoperitoneum under Spinal Anesthesia with Sedation Mahmoud Abo Amra Mahmoud ${ }^{1}$, Emad Adham Ibrahim1, Tareq Sobhey El-Naggar2, Mohammed Shaban Mohammed'.
}

\author{
Departments of General Surgery ${ }^{(1)}$ and Anesthesiology ${ }^{(2)}$, Faculty of Medicine Al-Azhar University (Assiut) ${ }^{1}$, \\ * Correspondences Author: Mohammed Shaban, Mobile:01090989358, \\ E-mail: medo_m18@ymail.com
}

\section{ABSTRACT}

Introduction: Cholecystectomy is the commonest operation of the biliary tract. It is known that open upper surgery under spinal anesthesia and laparoscopic cholecystectomy under general anesthesia.

Objective: In the present study study we performed laparoscopic cholecystectomy under spinal anesthesia with low tension pneumoperitoneum with sedation to evaluate, analyze and document whether laparoscopic cholecystectomy could be performed under spinal anesthesia and to assess the benefits and harms of low-tension pneumoperitoneum. The study was approved by the medical ethics committee of Al-Azhar University Hospital in Assiut and a written informed consent was obtained from all patients.

Materials and Methods: A prospective observational study with symptoms of cholelithiasis who underwent laparoscopic cholecystectomy under spinal anesthesia with low tension pneumoperitoneum (7-9 $\mathrm{mmHg}$ ) was conducted on 40 patients in Surgery Department of Al-Azhar University Hospital in Assiut from December 2018 to June 2019. Results: We successfully performed the operations in 27 patients without major complications. Only 13 patients $(32.5 \%)$ who converted to general anesthesia due to anxiety despite using sedation. The operation was converted to open cholecystectomy in one case only $(2.5 \%)$ due to disturbed anatomy and adhesions. Mean age was 45 years (range 21-57years). BMI was $\leq 32$. All patients were satisfied on follow up.

Conclusion: Laparoscopic cholecystectomy under spinal anesthesia with low-tension pneumoperitoneum with sedation by experienced surgeons is safe, cost-effective, feasible, associated with minimal postoperative pain and smooth recovery.

Keywords: Cholecystectomy, Laparoscopic cholecystectomy, Laparoscopy, Low tension pneumoperitoneum, Spinal anesthesia.

\section{INTRODUCTION}

Cholecystectomy is the commonest operation of the biliary tract. Laparoscopic cholecystectomy is considered the gold standard for therapy of symptomatic cholelithiasis. Laparoscopic cholecystectomy refers to minimally invasive procedure in which the gallbladder is removed through small incisions in the abdomen associated with less pain, reduced hospital stays, and earlier return to daily activities. Over 500,000 minimally invasive cholecystectomies are performed annually, with the majority being removed through a laparoscopic approach $^{(\mathbf{1})}$.

Creation of pneumoperitoneum (PP) by carbon dioxide $(\mathrm{CO} 2)$ insufflation is the most widely accepted technique for adequate working space and patient safety ${ }^{(2)}$.The standard pressure for PP lies between 12$15 \mathrm{~mm} \mathrm{Hg}$; however, decreased pulmonary compliance, altered blood gas parameters, decrease in cardiac output, impaired renal perfusion and raised liver enzymes have been observed at these pressures ${ }^{(3)}$. These effects may be explained by decrease in renal, hepato-portal and splanchnic blood flows, along with impairment of venous return during pneumoperitoneum. Several studies have compared the effects of reduced pressure $(7-9 \mathrm{~mm} \mathrm{Hg})$ with standard pressure $(12-15 \mathrm{~mm} \mathrm{Hg})$ during LC. These studies illustrate the feasibility of low-pressure PP, along with some advantages in terms of postoperative pain. However, it is still unclear whether changes in metabolic and physiologic parameters viz. liver function tests and cardiopulmonary parameters, have any clinical significance ${ }^{(\mathbf{1})}$.

General anesthesia is routinely used for this operation. To our knowledge, spinal anesthesia has not been reported in the medical literature as an anesthetic method for laparoscopic cholecystectomy. On the other hand, for open cholecystectomy, although general anesthesia is often used, spinal anesthesia has been infrequently reported as the anesthetic procedure of choice $^{(4)}$. Generally, spinal anesthesia was found to be associated with less post-operative mortality and other serious complications than general anesthesia ${ }^{(5)}$.

In some minor laparoscopic procedures, spinal anesthesia has been found to be associated with less emesis, less postoperative pain, shorter postoperative stay, better patient satisfaction, and improved overall safety ${ }^{(6)}$. Spinal anesthesia has been used with laparoscopic procedures such as diagnostic laparoscopy, infertility procedures, and tubal sterilization (7). Both laparoscopic inguinal hernia repair and laparoscopic appendicectomy have also been performed under spinal anesthesia ${ }^{(8)}$. However, for laparoscopic cholecystectomy, spinal anesthesia has not been reported as the sole anesthetic technique, but only as a conjunct to general anesthesia to increase the postoperative analgesic effect ${ }^{\left({ }^{(9)}\right.}$. In reviewing laparoscopic surgery, Motamed $\boldsymbol{e t}$ al. noted that all laparoscopic procedures are merely a change in access 
and still require general anesthesia, hence the difference from conventional surgery is likely to be small. Such a critical point of view, in our opinion, was one of the important factors that pushed many authors to try to prove that some laparoscopic procedures could be performed under regional anesthesia ${ }^{(8)}$. Because it has fewer postoperative complications than general anesthesia, spinal anesthesia seems more matching to the minimally invasive laparoscopic technique. We hoped to determine that laparoscopic cholecystectomy could be performed under spinal anesthesia with low tension pneumoperitoneum.

\section{AIM OF THE WORK}

To evaluate, analyze and document whether laparoscopic cholecystectomy could be performed under spinal anesthesia and to assess the benefits and harms of low-tension pneumoperitoneum.

\section{MATERIAL AND METHODS \\ Patients:}

This prospective observational study will be conducted in Surgery Department of Al-Azhar University Hospital in Assiut from December 2018 to June 2019. Forty patients with symptoms of cholelithiasis were selected randomly by closed envelope technique. Their age ranged between 22 to 68 years and were considered eligible to laparoscopic cholecystectomy with low tension pneumoperitoneum under spinal anesthesia. Informed consent was obtained from each patient. Patients were offered the possibility of conversion to general anesthesia if they were unsatisfied with spinal anesthesia at any time during the procedure .

Inclusion criteria of the patients were: Age (2268 ) years, both sexes, ASA class (I or II), BMI $\leq 32$, Normal coagulation profile.

Exclusion criteria of the patients were: Patients below 22 years and above 68 years. Patients with acute cholecystitis, pancreatitis, peritonitis or cholangitis, biliary obstruction, prior laparotomy for upper abdominal surgery. Patients with cancer head of pancreas, with hepatitis B, C, with history of malignancy, history of alcohol intake and history of jaundice, contraindication for pneumoperitoneum, contraindication for spinal anesthesia owing to spinal deformity. Severe cardiorespiratory in sufficiency, bleeding diathesis, chronic debilitating disease (liver cell failure, chronic renal failure).

All patients involved in the present study were subjected to full clinical assessment and required routine investigations: pelviabdominal ultrasound, coagulation profile, fasting blood sugar, liver function tests, kidney function tests, hepatitis marker, complete blood count, ECG and chest x-ray. Patients are fasted for a minimum of 8 hours prior to the operation. Prophylactic antibiotics are up to the surgeon's discretion; evidence suggests that most patients have a very low risk of perioperative infection. Anti-embolic stockings and sequential compression devices are placed on both legs to avoid pooling of blood in the lower extremities by the reverse Trendelenburg position generally used during this operation. An orogastric tube may be placed to decompress the stomach. The abdomen is shaved and prepared in standard sterile fashion with particular care taken to rid the umbilicus of all debris.

Evaluation of operative time, blood loss and any intra operative complication: biliary injury, intraperitoneal hemorrhage, missed CBD stone, seroma, wound infection, post-operative acute pain, chronic pain, ability of patients to walk, postoperative hospital stays, intestinal obstruction.

\section{Ethical consideration and written informed consent:}

The study was approved by the medical ethics committee of Al-Azhar University Hospital in Assiut and a written informed consent was obtained from all patients.

\section{Methods:}

Positioning: Place the patient in the supine position. Place peripheral intravenous lines, along with ECG pulse oximetry, and blood pressure monitors.

Anesthesia: All patients were monitored for noninvasive blood pressure, oxygen saturation, and heart rate just before the operation. Intravenous catheterization was done with a 16-Gcatheter inserted in the left hand, and $500 \mathrm{~mL}$ ringer lactate was infused to all patients. After recording the vital parameters, all patients were brought to a sitting position. A 26-G spinal needle was introduced to the intrathecal space between lumbar 1 and 2 interspaces. Spinal anesthetic agents were given to this space after free flow of clear cerebrospinal fluid was observed. The agent for spinal anesthesia was ropivacaine and bupivacaine. Also, fentanyl as an adjunct to local anesthetic drugs at a dosage of $25 \mathrm{mg}$ was given to all patients intrathecally. The patients were then placed into a semi Fowler position at an angle of 30 to 45 degrees. Pinprick test was performed to evaluate the sensory block level, which was accepted as T4 dermatome level to allow LC. As soon as the sensory block level reached T4 dermatome level, the procedure was begun. Blood pressure, oxygen saturation, and heart rate were measured and recorded during the operation on all patients. If mean blood pressure was lower than $60 \mathrm{~mm}$ $\mathrm{Hg}$, ephedrine (10 mg intravenously) was administered. fentanyl (25mg intravenously) was given if patients complained about severe shoulder pain.

\section{Operative technique}

After spinal puncture, the skin was prepared initially with chlorhexidine from just below nipple line to the inguinal ligaments and laterally to the anterior 
iliac spine. Then the operative field was draped. A 1.5$\mathrm{cm}$ longitudinal incision at the inferior aspect of umbilicus was made and it was deepening through the subcutaneous fat to the rectus sheath. With a Kocher clamp, the reflection of the linea Alba was grasped and elevated into the umbilicus.

The peritoneum was elevated between 2 straight clamps and incised, affording safe entry into the abdominal cavity. An 11-mm blunt Hasson trocar was placed into the abdominal cavity and initiate $\mathrm{CO} 2$ insufflation to pressure between 9-12 mm Hg. A 30degree laparoscopic is preferred because it gives better visualization of the cystic structures from multiple vantage points and requires a more skilled scope operator. The laparoscope was advanced slowly into the abdominal cavity.

An incision 3 finger breadths below the xiphoid process was made and deeping into the subcutaneous fat. An $10 \mathrm{~mm}$ trocar was advanced into the abdominal cavity (under direct vision) in the direction of the gallbladder through the abdominal wall and entered to the right of the falciform ligament. The table was placed in reverse Trendelenburg position with the right side up to allow the small bowel and colon to fall away from the operative field). Intra-abdominal pressure was set at $8 \mathrm{~mm}$ of $\mathrm{Hg}$. Pneumoperitoneum was started at the rate of $11 / \mathrm{min}$. A grasper was used to retract the fundus of the gallbladder laterally and anteriorly through the most lateral subcostal cannula. The cystic duct and artery were isolated, closed and divided using metal clips and scissors. Then the gall bladder was dissected from liver bed and delivered through the subxiphoid incision. After that the table was returned to neutral position.

The gallbladder bed and the suprahepatic spaces were irrigated and sucked to ensure adequate homeostasis and removal of any debris or bile that may have spilled. Under direct vision, the subxiphoid and 2 5 -mm ports were removed, following trocar. The facia was closed at the umbilical port using the $2 \mathrm{U}$-stitches places at the procedure. At the completion of the previously port umbilical closure was placed using $\mathrm{U}$ stitches. All the skin incisions were closed with 4-0 absorbable suture, followed by Derma bond. In cases of elective cholecystectomy, the patient can be discharged home with combination acetaminophen/opiate oral pain medication.

\section{Post-operative follows up:}

The patients were discharged one day postoperative and were followed up at outpatient clinic after one and two weeks. Early post-operative follows up involved assessment of the course and complication including as bleeding, infection biliary leakage, pneumothorax subcutaneous emphysema, and pneumomediastinum, Carbon Dioxide Gas Embolism.

\section{Statistical Analysis}

The data were tested for normality using the Anderson-Darling test and for homogeneity variances prior to further statistical analysis. Categorical variables were described by number and percent $(\mathrm{N}$, $\%)$, where continuous variables described by mean and standard deviation (Mean, SD). Chi-square test and fisher exact test used to compare between categorical variables where compare between continuous variables by t-test. A two-tailed $\mathrm{p}<0.05$ was performed with the IBM SPSS 20.0 software.

\section{RESULTS}

This prospective randomized study was conducted at Al-Azhar University hospital, Assiut between from December 2018 to June 2019 and included 40 patients with gall bladder diseases.

1- Patient age:

Table (1): Age distribution.

\begin{tabular}{|c|c|c|c|c|}
\hline \multirow{2}{*}{$\begin{array}{l}\text { Mean } \\
\text { (Years) }\end{array}$} & \multirow{2}{*}{$\begin{array}{l}\text { Standard } \\
\text { Deviation }\end{array}$} & $\operatorname{Max}$ & Min & \multirow[t]{2}{*}{$\mathrm{P}$} \\
\hline & & \multicolumn{2}{|c|}{ Years } & \\
\hline 45.0 & 11.18 & 57 & 21 & 0.06 \\
\hline
\end{tabular}

2)Patients gender:

Table (2): Sex distribution

\begin{tabular}{|c|c|c|}
\hline Males & Females & Total \\
\hline $\mathbf{4 ( 1 0 \% )}$ & $36(90 \%)$ & $\mathbf{4 0}(\mathbf{1 0 0 \% )}$ \\
\hline
\end{tabular}

3) BMI (Body mass index):

Table (3): BMI

\begin{tabular}{|c|c|c|}
\hline BMI & Number & Percentage \\
\hline$<20$ & $\mathbf{4}$ & $\mathbf{1 0 \%}$ \\
\hline $20-25$ & $\mathbf{2 3}$ & $\mathbf{5 7 . 5 \%}$ \\
\hline $25-30$ & $\mathbf{1 0}$ & $\mathbf{2 5 \%}$ \\
\hline$>\mathbf{3 0}$ & $\mathbf{3}$ & $\mathbf{7 . 5 \%}$ \\
\hline
\end{tabular}

4) Associated co-morbidity:

Table (4): Associated comorbidity

\begin{tabular}{|c|c|c|}
\hline D.M & 7 & $17.5 \%$ \\
\hline Hypertension & 5 & $12.5 \%$ \\
\hline Bronchial asthma & 2 & $5 \%$ \\
\hline I.H. D & 1 & $2.5 \%$ \\
\hline Atherosclerosis & 1 & $2.5 \%$ \\
\hline
\end{tabular}

5) Duration of operative procedures (in minutes).

Table (5): Duration of operative procedure

\begin{tabular}{|c|c|l|l|l|}
\hline $\begin{array}{c}\text { Mean } \\
\text { minutes) }\end{array}$ & Standard Deviation & \multicolumn{2}{|l|}{ Min Max } & P \\
\cline { 2 - 4 } & & Minutes & \\
\hline 55 & \pm 15 & 40 & 70 & 0.235 \\
\hline
\end{tabular}


6) Conversion rate for general anesthesia:

Patients were offered the possibility of conversion to general anesthesia if they were unsatisfied with spinal anesthesia at any time during the procedure.

Number of cases who convert to general anaesthesia 13 cases (32.5\%) from 40 cases and 27 cases complete under spinal anesthesia $(67.5 \%)$.

\section{Conversion rate}

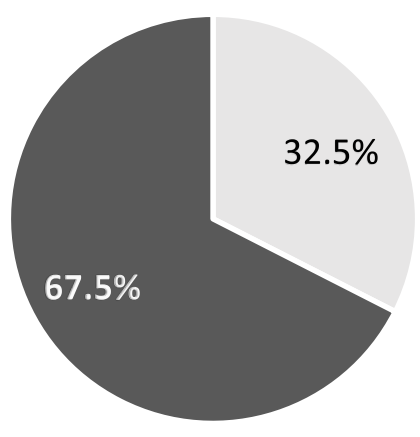

Conversion rate $\quad$ Complete under spinal

Fig (1): Pei charts showing conversion rate.

\section{7) Post-operative pain:}

It was evaluated at 12,24,48 hours after operation using a numeric pain scoring system. According to PIC score the pain ranges from 2-4 degrees.

Table (6): Pain degree

\begin{tabular}{|c|c|c|c|c|}
\hline \multirow{2}{*}{$\begin{array}{c}\text { Mean } \\
\text { degrees })\end{array}$} & \multirow{2}{*}{$\begin{array}{l}\text { Standard } \\
\text { Deviation }\end{array}$} & Min & Max & P \\
\cline { 3 - 4 } & & \multicolumn{2}{|c|}{ Degrees } & \\
\hline $\mathbf{3}$ & $\pm \mathbf{1}$ & $\mathbf{2}$ & $\mathbf{4}$ & $\mathbf{0 . 3 2 6}$ \\
\hline
\end{tabular}

The patients who need analgesia after 1.5 to 2.5 hours.

Table (7): Doses of Analgesia

\begin{tabular}{|c|c|c|}
\hline $\begin{array}{c}\text { Analgesia } \\
\text { Number of additional doses of } \\
\text { analgesic }\end{array}$ & 5 & 35 \\
\hline Total extra dose of analgesic & 12 & 28 \\
\hline
\end{tabular}

8) Lengths of hospital stay (in days).

Table (8): Length of hospital stay

\begin{tabular}{|c|c|c|c|c|}
\hline \multirow{2}{*}{$\begin{array}{c}\text { Mean } \\
\text { (Days) }\end{array}$} & $\begin{array}{l}\text { Standard } \\
\text { Deviation }\end{array}$ & Min & Max & P \\
\cline { 3 - 4 } & & \multicolumn{2}{|c|}{ Days } & \\
\hline $\mathbf{1 . 5}$ & 0.5 & $\mathbf{1}$ & $\mathbf{2}$ & $\mathbf{0 . 2 4 5}$ \\
\hline
\end{tabular}

9) Duration of return to work. (In days).

Table (9): Duration of return to work

\begin{tabular}{|c|c|c|c|c|}
\hline \multirow{2}{*}{$\begin{array}{l}\text { Mean } \\
\text { (Days) }\end{array}$} & \multirow{2}{*}{$\begin{array}{l}\text { Standard } \\
\text { Deviation }\end{array}$} & Min & Max & \multirow[t]{2}{*}{$\mathbf{P}$} \\
\hline & & \multicolumn{2}{|c|}{ Days } & \\
\hline 8.5 & 1.5 & 7 & 10 & 0.225 \\
\hline
\end{tabular}

10) Post-operative complications.

Table (10): Post-operative complications.

\begin{tabular}{|c|c|}
\hline Complications & $\begin{array}{l}\text { Number of } \\
\text { patients }\end{array}$ \\
\hline \multicolumn{2}{|l|}{ Perioperative complication: } \\
\hline Bleeding & $2(5 \%)$ \\
\hline Biliary injury & 0 \\
\hline Missed CBD stone & 0 \\
\hline \multicolumn{2}{|l|}{ Postoperative complication: } \\
\hline Seroma & $1(2.5 \%)$ \\
\hline Wound infection & 0 \\
\hline Chronic abdominal pain & $2(5 \%)$ \\
\hline \multicolumn{2}{|l|}{ Anesthetic complication: } \\
\hline -Post spinal headache. & $5(12.5 \%)$ \\
\hline -Urine retention. & $2(5 \%)$ \\
\hline -Nausea \&Vomiting. & $8(20 \%)$ \\
\hline - Shoulder pain & $12(30 \%)$ \\
\hline -Anxiety & $13(32.5 \%)$ \\
\hline $\begin{array}{l}\text { - Stomach distension requiring } \\
\text { Ryle's tube aspiration }\end{array}$ & $3(7.5 \%)$ \\
\hline
\end{tabular}

\section{1) Follow up period.}

Table (11): Follow up period.

\begin{tabular}{|c|c|c|c|c|}
\hline \multirow{2}{*}{$\begin{array}{c}\text { Mean } \\
\text { (Days) }\end{array}$} & $\begin{array}{l}\text { Standard } \\
\text { Deviation }\end{array}$ & Min & Max & P \\
\cline { 3 - 5 } Days & \\
\hline $\mathbf{9 . 5}$ & 2.5 & $\mathbf{7}$ & $\mathbf{1 2}$ & $\mathbf{0 . 2 3 8}$ \\
\hline
\end{tabular}

\section{DISCUSSION}

Laparoscopic cholecystectomy is the gold standard treatment for gall stones. Gallstone disease is a frequent medical problem. Gallstones affects $10 \%$ of the population and $30 \%$ of patients with gallstones will undergo surgery. During the last two decades, the general principles of gallstone management have not notably changed. However, methods of treatment have been dramatically altered. Today, laparoscopic cholecystectomy, laparoscopic common bile duct exploration, and endoscopic retrograde management of common bile duct (CBD) stones play important roles in the treatment of gallstones ${ }^{(\mathbf{1 0})}$.

Spinal anesthesia can be an easier technique than general anesthesia ${ }^{(\mathbf{1 1})}$. Monitoring of patients under spinal anesthesia is easier than general anesthesia. Complication of endotracheal intubations like damage to oral cavity, teeth, sore throat, aspirations and failure of intubations are absent in spinal anesthesia. Cost of spinal anesthesia is less than general anesthesia and nausea and vomiting are less with spinal anesthesia ${ }^{(\mathbf{1 2})}$.

Laparoscopic cholecystectomy with low-pressure pneumoperitoneum under spinal anesthesia is effective in patients with COPD, who are unfit for general anesthesia ${ }^{(13,14)}$. 
In the present study, we randomly select 40 patients for 6 months with symptomatic cholelithiasis, suitable inclusion criteria, their age ranged from 22 to 57 years, (90\%) of them are females, BMI is less than 32. All worked up for laparoscopic cholecystectomy under spinal anaesthesia with low pressure pneumoperitoneum (LPLC) ranged between 7 to 9 $\mathrm{mmHg}$. We didn't find any difficulties in placement of any port except slightly the 2 nd port. We found that the operative time in laparoscopic cholecystectomy ranged between 40 to 70 minutes (Mean \pm SD 55 \pm 15 ). The rate of conversion from spinal to general anesthesia is $32.5 \%$ (13 cases) and 27 cases complete the operation under spinal anesthesia $(67.5 \%)$. The rate of conversion from laparoscopic to open cholecystectomy is $2.5 \%$ (1case only) due to difficult in dissection with adhesion. The results in our study show that the pain scores in the operative day and first postoperative week decreased progressively in all patients. According to PIC score, in our study, pain scores ranged between 24 degrees (Mean \pm SD $3 \pm 1$ ). There was no wide individual variation of pain scores. The number of additional doses of analgesia were 5 patients who used additional doses of analgesia post-operative $(12.5 \%)$ after two and half hour of operation and total extra dose of analgesics. Tweleve patients needed for extra dose of analgesia (30\%) after five hours of operation. Intraoperative bleeding occured in two cases only and management of bleeding was done.

According to post-operative complications, seroma was occurred in one case only and there was no wound infection due to good sterilization. Only two cases recorded chronic pain. Regarding shoulder pain during and post-operative: the results show that twelve cases $(30 \%)$ only complained from pain which relieved with sedation and analgesia.

Only eight patients $(20 \%)$ had post-operative nausea and vomiting and relived by antiemetic drugs. Also, thirteen patients $(32.5 \%)$ had anxiety and relived by sedations which explain conversion to GA. Stomach distension which required Ryle's tube aspiration to decrease the distention was occurred in three patients only $(7.5 \%)$.

Urine retention was occurred in two patients (5\%) who need urinary catheterization.

The length of hospital stays ranged between 1-2 days (Mean \pm SD1.5 \pm 0.5 ) and there was no statistically significant difference. According to duration of intolerability of work, the patients returned to their work between 7-10 days $(8.5 \pm 1.5)$ with a follow up of 7-12 days after discharge.

\section{CONCLUSION}

Laparoscopic cholecystectomy under spinal anesthesia with low-pressure pneumoperitoneum with sedation by experienced surgeons is safe, costeffective, feasible, associated with minimal postoperative pain and smooth recovery. The most important advantage of spinal anesthesia is making laparoscopic surgery possible, even in settings where facilities for general anesthesia may not be available.

\section{REFERENCES}

1. Lichten JB, Reid JJ, Zahalsky MP et al. (2001): Laparoscopic cholecystectomy in the new millennium. Surg Endosc., 15:867-872.

2. Gurusamy K, Junnarkar S, Farouk M et al. (2012): Analysis of randomized controlled trials on the effectiveness of day-case laparoscopic Surge., 95:161-8.

3. Henny CP, Hofland J (2005): Laparoscopic surgery: pitfalls due to anesthesia, positioning, and pneumoperitoneum. Surg Endosc., 19 (9):1163-1171

4. Ajao OG, Adeloya A (2007): The importance of spinal anesthesia in surgical practice in tropical Africa. J Trop Med Hyg., 80: 126-128.

5. Racle JP, Benkhadra A, Poy JY et al. (1998): Comparative study of general and spinal anesthesia in elderly women in hip surgery. Ann Fr Anesth Reanim., 5: 24-30.

6. Collins LM, Vaghadia H (2001): Regional anesthesia for laparoscopy. Anesthesiol Clin North Am., 19: 43-5.

7. Peck EM (2001): Laparoscopic tubal sterilization under spinal anesthesia in the third world. Modern Med., 98: 10-11.

8. Inoue H, Takeshita K, Endo M (2003): Single-port laparoscopy assisted appendectomy under local pneumoperitoneum condition. Surg Endosc., 8: 714-71.

9. Motamed C, Bouaziz H, Franco D et al. (2000): Analgesic effect of low-dose intrathecal morphine and bupivacaine in laparoscopic cholecystectomy. Anesthesia, 55: 118-124.

10. Haydon GH, Dillon J, Simpson KJ et al. (1996): Hypoxemia during diagnostic laparoscopy: a prospective study. Gastrointest Endosc., 44(2):124 - 8.

11. Shamiyeh A, Wayand W (2006): Current Status of laparoscopic therapy of cholelithiasis and common bile duct stones Dig Dis., 23:119-26.

12. Yuksek YN, Akat AZ, Gozalan U et al. (2008): Laparoscopic cholecystectomy under spinal anesthesia. Am J Surg., 195:533-6.

13. Barczynski M, Herman RM (2003): A prospective randomized trial on comparison of low pressure (LP) and standard pressure (SP) pneumoperitoneum for laparoscopic cholecystectomy. Surg Endosc., 17:533-8.

14. Van Zundert AA, Stultiens G, Jakimowicz JJ et al. (2006): Segmental spinal anesthesia for cholecystectomy in a patient with severe lungs disease. $\mathrm{Br} \mathrm{J}$ Anaesth., 96:464-6. 